\title{
A POLYNOMIAL TIME ALGORITHM FOR GEODETIC HULL NUMBER FOR COMPLEMENTARY PRISMS*
}

\author{
Erika M.M. Coelho ${ }^{1}$, Hebert Coelho ${ }^{1}$, Julliano R. Nascimento ${ }^{1, * *} \odot$ \\ AND JAYME L. SZWARCFITER ${ }^{2,3}$
}

\begin{abstract}
Let $G$ be a finite, simple, and undirected graph and let $S \subseteq V(G)$. In the geodetic convexity, $S$ is convex if all vertices belonging to any shortest path between two vertices of $S$ lie in $S$. The convex hull $H(S)$ of $S$ is the smallest convex set containing $S$. The hull number $h(G)$ is the minimum cardinality of a set $S \subseteq V(G)$ such that $H(S)=V(G)$. The complementary prism $G \bar{G}$ of a graph $G$ arises from the disjoint union of the graph $G$ and $\bar{G}$ by adding the edges of a perfect matching between the corresponding vertices of $G$ and $\bar{G}$. Previous works have determined $h(G \bar{G})$ when both $G$ and $\bar{G}$ are connected and partially when $G$ is disconnected. In this paper, we characterize convex sets in $G \bar{G}$ and we present equalities and tight lower and upper bounds for $h(G \bar{G})$. This fills a gap in the literature and allows us to show that $h(G \bar{G})$ can be determined in polynomial time, for any graph $G$.
\end{abstract}

Mathematics Subject Classification. 05C69, 05C76, $05 \mathrm{C} 85$.

Received August 3, 2021. Accepted January 4, 2022.

\section{INTRODUCTION}

In this paper we consider finite, simple, and undirected graphs. For a graph $G$ with vertex set $V(G)$, a graph convexity on $V(G)$ is a collection $\mathcal{C}$ of subsets of $V(G)$ such that $\emptyset, V(G) \in \mathcal{C}$ and $\mathcal{C}$ is closed under intersections. The sets in $\mathcal{C}$ are called convex sets and the convex hull $H(S)$ of a set $S \subseteq V(G)$ is the smallest set in $\mathcal{C}$ containing $S$.

In recent decades, the concepts of graph convexity have received attention due their versatility for modelling problems in contexts that requires some disseminating process, such as contamination [6], marketing strategies [14], spread of opinion [17], and distributed computing [26].

In this paper, we are concerned with the convexity related to shortest paths in graphs, the geodetic convexity $\mathcal{C}$. Given a graph $G$, the closed interval $I[u, v]$ of a pair $u, v \in V(G)$ consists of $u, v$, and all vertices lying in any shortest path between $u$ and $v$ in $G$. For a set $S \subseteq V(G)$, the closed interval $I[S]$ is the union of all sets $I[u, v]$ for $u, v \in S$. If $I[S]=S$, then $S$ is a convex set. If $H(S)=V(G)$ we say that $S$ is a (geodetic) hull set of $G$. The cardinality $h(G)$ of a minimum hull set of $G$ is called the (geodetic) hull number of $G$.

\footnotetext{
* The authors are partially supported by CAPES, CNPq, and FAPERJ.

Keywords and phrases: Complementary prisms, geodetic convexity, hull number.

${ }^{1}$ INF, Universidade Federal de Goiás, GO, Brazil.

2 IM, COPPE, and NCE, Universidade Federal do Rio de Janeiro, RJ, Brazil.

3 IME, Universidade do Estado do Rio de Janeiro, RJ, Brazil.

** Corresponding author: jullianonascimento@inf.ufg.br
} 
Given a graph $G$ and an integer $k$, the problem of deciding whether $h(G) \leq k$ is NP-complete for a general graph $G$, even if $G$ is bipartite [3], partial cube [2], chordal [5], or $P_{9}$-free [16]. On the other hand, such a parameter can be determined in polynomial time for unit interval graphs, cographs, split graphs [15], $(q, q-4)$ graphs [3], \{paw, $\left.P_{5}\right\}$-free graphs [4, 16], and distance-hereditary graphs [24]. Coelho et al. [12] provide additional references concerning the hull number, also in other graph convexities.

Let $G$ be a graph and $\bar{G}$ its complement. For a vertex $v \in V(G)$ we denote $\bar{v} \in V(\bar{G})$ as its corresponding vertex. For a set $X \subseteq V(G)$, we call $\bar{X}=\{\bar{v} \in V(\bar{G}): \bar{v}$ is corresponding to $v \in X\}$ the corresponding set of $X$ in $V(\bar{G})$. The complementary prism $G \bar{G}$ of $G$ arises from the disjoint union of $G$ and its complement $\bar{G}$ by adding the edges of a perfect matching joining corresponding vertices of $G$ and $\bar{G}$ [23]. We say that a graph $G$ is autoconnected if both $G$ and $\bar{G}$ are connected.

It is known that the hull number for complementary prisms $G \bar{G}$ can be determined in polynomial time in the $P_{3}$-convexity [18]. In fact, such parameter is equal to $t+1$ when $G$ has $t \geq 2$ connected components [18] and it is limited to 3 when $G$ is autoconnected [7]. Related to the geodetic convexity, in 2018, a preliminary work on the hull number for complementary prisms $G \bar{G}$ showed bounds when $G$ is a tree, a disconnected graph or a cograph [11]. Later, Castonguay et al. [9] study the hull number for complementary prisms $G \bar{G}$ providing results for any autoconnected graph $G$.

A well-known subclass of autoconnected graphs is the class of selfcomplementary graphs, i.e., graphs that are isomorphic to their complements [20]. Moreover, there is a series of papers by Akiyama and Harary surveying the structure of graphs $G$ such that both $G$ and $\bar{G}$ possess some property. The first of them [1] exploit conditions to a graph to be autoconnected. Furthermore, some graph parameters has been studied for autoconnected graphs such as connected domination number [25] and Nordhaus-Gaddum problems for diameter, girth, circumference and edge covering number [27].

A split graph $G$ is one whose vertex set admits a partition $V(G)=C \cup I$ into a clique $C$ and an independent set $I$. Particularly, when dealing with split graphs, Castonguay et al. [9] use the following definitions.

Definition 1.1 (Castonguay et al. [9]). Let $G=(C \cup I, E)$ be a split graph. We define the component graph $G^{\prime}$ of $G$ as

$$
G^{\prime}=G \backslash E(G[C])=\bigcup_{i=1}^{c\left(G^{\prime}\right)} G_{i}
$$

in which $c\left(G^{\prime}\right)$ denotes the number of connected components of $G^{\prime}$.

Notice that in Definition 1.1, the graphs $G_{i}$, for every $i \in\left\{1, \ldots, c\left(G^{\prime}\right)\right\}$, are the connected components of $G^{\prime}$.

Definition 1.2 (Castonguay et al. [9]). Let $G=(C \cup I, E)$ be a split autoconnected graph. We define the set $L\left(G_{i}\right)$ of a component $G_{i}$ of $G^{\prime}$ as

$$
L\left(G_{i}\right)=V\left(G_{i}\right) \cup\left(V\left(\bar{G}_{i}\right) \cap \bar{C}\right),
$$

for every $i \in\left\{1, \ldots, c\left(G^{\prime}\right)\right\}$.

In this work, we show that Definition 1.2 can be easily adapted for disconnected split graphs. This allows us to prove the equality for the case that was not completely settled by [11], which is the case $G$ is a disconnected graph with exactly one nontrivial component $H$. Particularly, the bounds presented in [11] for $h(G \bar{G})$ in terms of $h(H)$ can be far from sharpness (see a comparison in Rem. 3.14). The bounds obtained in this paper are tight and the equalities generalize those in [11].

Furthermore, we answer an open question left by [11] regarding the complexity of the hull number decision problem for complementary prisms. By putting together the results presented in this paper and the ones by 
Castonguay et al. [9], we show how to determine the hull number of any complementary prism $G \bar{G}$ in $O\left(n^{6}\right)$ time, for $n=|V(G \bar{G})|$.

This paper is organized in more three sections. In Section 2 we define the fundamental concepts. In Section 3 we present our results. We close with some further remarks in Section 4.

\section{PRELIMINARIES}

Let $G$ be a graph. We denote by $N_{G}(v)\left(N_{G}(U)\right)$ the open neighborhood of a vertex $v \in V(G)$ (a set $U \subseteq V(G)$ ), and by $N_{G}[v]$ the closed neighborhood of $v \in V(G)$. A clique of a graph $G$ is a subset of pairwise adjacent vertices in $G$. We say that $v$ is a simplicial vertex of $G$ if $N_{G}[v]$ induces a clique. We denote by $\sigma(G)$ the set of all simplicial vertices of a graph $G$. The set of positive integers $\{1, \ldots, k\}$ is denoted by $[k]$.

We denote by $d_{G}(u, v)$ the distance between $u, v \in V(G)$, by $\operatorname{diam}(G)$ the diameter of a graph $G$ and by $\operatorname{deg}_{G}(u)$ the degree of a vertex $u \in V(G)$. A graph $G$ is called connected if any two of its vertices are linked by a path in $G$. Otherwise, $G$ is called disconnected. A maximal connected subgraph of $G$ is called a (connected) component of $G$. A connected component $G^{\prime}$ of a graph $G$ is trivial, if $\left|V\left(G^{\prime}\right)\right|=1$, and nontrivial otherwise.

Two graphs $G=(V, E)$ and $G^{\prime}=\left(V^{\prime}, E^{\prime}\right)$ are isomorphic if and only if there is a bijection $\varphi: V \rightarrow V^{\prime}$ such that $u v \in E$ if and only if $\varphi(u) \varphi(v) \in E^{\prime}$, for every $u, v \in V$. We denote by $G \simeq G^{\prime}$, if $G$ and $G^{\prime}$ are isomorphic, and by $G \not G^{\prime}$, otherwise.

Throughout this paper, if $G$ is a split graph, we consider the partition of $V(G)=C \cup I$ such that $C$ is a maximum clique.

\section{Computing the hull Number}

We begin by enunciating three useful lemmas.

Lemma 3.1 (Everett and Seidman [19]). For every hull set $S$ of a graph $G, S$ contains $\sigma(G)$.

Lemma 3.2 (Dourado et al. [15]). Let $G$ be a graph and $S$ a proper and nonempty subset of $V(G)$. If $V(G) \backslash S$ is convex then every hull set of $G$ contains at least one vertex of $S$.

Lemma 3.3. Let $G \bar{G}$ be a complementary prism, for some graph $G$. If $u \in \sigma(G)$ and $\bar{u} \in \sigma(\bar{G})$, then every hull set $S$ of $G \bar{G}$ intersects $\{u, \bar{u}\}$.

Proof. Suppose, by contradiction, that there exists a hull set $S$ of $G \bar{G}$ such that $S \cap\{u, \bar{u}\}=\emptyset$.

Since $S$ is a hull set of $G \bar{G}$, we have that $u, \bar{u} \in H(S)$. Let $u \in I[x, y]$, for $x, y \in V(G) \backslash\{u\}$. Since $u$ is simplicial in $G$, we have that any $(x, y)$-path $P$ which passes through $u$ contain $w, w^{\prime} \in N_{G}(u)$. Since $u \in \sigma(G)$, $w w^{\prime} \in E(G)$, then $P$ is not a shortest path, a contradiction. Similarly, it is possible to show that $\bar{u} \notin I[\bar{x}, \bar{y}]$, for every $\bar{x}, \bar{y} \in V(\bar{G}) \backslash\{\bar{u}\}$. Then, we assume that $u, \bar{u} \in I[x, \bar{y}]$, for $x \in V(G) \backslash\{u\}$ and $\bar{y} \in V(\bar{G}) \backslash\{\bar{u}\}$.

By definition of complementary prism, for every $x, y \in V(G \bar{G}) \backslash\{u, \bar{u}\}$, a $(x, y)$-path $P$ containing $u, \bar{u}$ has length at least 3 . Clearly, since $d_{G \bar{G}}(x, y)=2, P$ is not a shortest path, a contradiction.

An alternative proof of Lemma 3.3 appears in [11].

Clearly, Lemma 3.3 provides a lower bound for $h(G \bar{G})$ that is the number of simplicial vertices $u \in V(G)$ such that its corresponding vertex $\bar{u}$ is simplicial in $\bar{G}$. Before we discuss our lower and upper bounds, we show two auxiliary propositions.

Proposition 3.4 (Castonguay et al. [9]). Let $G$ be a graph, $S \subseteq V(G \bar{G})$, and $v_{1} \ldots v_{k}$ be a path in $G$, for $k \geq 2$. If $\left\{v_{1}, \bar{v}_{2}, \ldots, \bar{v}_{k}\right\} \subseteq H(S)$, then $v_{k} \in H(S)$.

Proposition 3.5. Let $G$ be a graph and uxyv an induced $P_{4}$ in $G$. If $u, v \subseteq H(S)$, then $\{u, x, y, v, \bar{u}, \bar{x}, \bar{y}, \bar{v}\} \subseteq$ $H(S)$. 
Proof. By definition of complementary prism and by hypothesis, respectively, uñuv $v$ and uxyv are shortest paths between $u$ and $v$. Then $u, x, y, v, \bar{u}, \bar{v} \in I[u, v]$. Since $u y, v x \notin E(G)$, we have that $\bar{u} \bar{y}, \bar{v} \bar{x} \in E(\bar{G})$. Consequently $\bar{x} \in I[x, \bar{v}], \bar{y} \in I[y, \bar{u}]$, and the conclusion follows.

Similarly as done in [9] for autoconnected graphs $G$, our results are divided considering $G$ a split graph or not. But here we are concerned with disconnected graphs $G$. Since either $G$ or $\bar{G}$ is connected, we assume from now that $G$ is a disconnected graph.

\subsection{Nonsplit graphs}

We show in this section our results for nonsplit disconnected graphs.

Theorem 3.6. Let $G$ be a nonsplit graph with $t \geq 2$ connected components. Then $t+1 \leq h(G \bar{G}) \leq t+2$.

Proof. Let $G$ be a nonsplit graph with connected components $G_{1}, \ldots, G_{t}$, for some $t \geq 2$, and $S$ a hull set of $G \bar{G}$. Since $G$ is disconnected, then, for every pair $\bar{u}, \bar{v} \in V(\bar{G}), d_{G \bar{G}}(\bar{u}, \bar{v}) \leq 2$. This implies that, for every shortest $(\bar{u}, \bar{v})$-path $P, P \cap V(G)=\emptyset$. Thus, if $S \cap V\left(G_{i}\right)=\emptyset$, for some $i \in[t]$, then $H(S) \cap V\left(G_{i}\right)=\emptyset$, which implies that $h(G \bar{G}) \geq t$. We show that $h(G \bar{G}) \geq t+1$.

Let $S$ be a hull set of $G \bar{G}$ such that $\left|S \cap V\left(G_{i}\right)\right|=1$, for every $i \in[t]$. Since $G \not \bar{K}_{t}$, there exists $i \in[t]$ such that $\left|V\left(G_{i}\right)\right| \geq 2$. It is clear that $H(S)=S \cup \bar{S}$, then $S$ is not a hull set of $G \bar{G}$. Hence, $h(G \bar{G}) \geq t+1$.

For the upper bound, we show hull sets of $G \bar{G}$ of order $t+1$ and $t+2$. Since $G$ is a nonsplit graph, we consider that $G$ has a $C_{4}, C_{5}$ or a $2 K_{2}$ as an induced subgraph [21].

For every $i \in[t]$, we denote $V\left(G_{i}\right)=\left\{v_{1}^{i}, \ldots, v_{\mid V\left(G_{i} \mid\right.}^{i}\right\}$. Let $F \in\left\{C_{4}, C_{5}, 2 K_{2}\right\}$ be an induced subgraph of $G$. It is clear that, if $F \in\left\{C_{4}, C_{5}\right\}$, then $F \subseteq G_{i}$, for some $i \in[t]$. Otherwise, $F \simeq 2 K_{2}$ implies two cases, either $F \subseteq G_{i}$ or $\left(V(F) \cap V\left(G_{i}\right) \neq \emptyset\right.$ and $\left.V(F) \cap V\left(G_{j}\right) \neq \emptyset\right)$, for some $i, j \in[t]$. Thus, w.l.o.g., we assume that $F \subseteq G_{1} \cup G_{2}$, and we distinguish two cases: $F \subseteq G_{1}$ and $\left(V(F) \cap V\left(G_{1}\right) \neq \emptyset\right.$ and $\left.V(F) \cap V\left(G_{2}\right) \neq \emptyset\right)$. Let $x, y, z \in V(F)$ such that $x y \in E(F)$ and $y z \notin E(F)$. We define $S_{1}=\left\{x, y, z, v_{1}^{2}, \ldots, v_{1}^{t}\right\}$ for the former and $S_{2}=\left\{x, y, z=v_{1}^{2}, v_{1}^{3}, \ldots, v_{1}^{t}\right\}$ for the latter. Notice that $\left|S_{1}\right|=t+2$ and $\left|S_{2}\right|=t+1$. We show that $S \in\left\{S_{1}, S_{2}\right\}$ is a hull set of $G \bar{G}$, by proving first that $V(F) \cup V(\bar{F}) \subseteq H(S)$.

Since, $\bar{u}, \bar{v}_{1}^{t}$ lie in a shortest $\left(u, v_{1}^{t}\right)$-path, for every $v \in S \backslash\left\{v_{1}^{t}\right\}$, we have that $\bar{S} \subseteq I[S]$. If $V(F)=\{x, y, z, w\}$ induces a $C_{4}$ (resp. $\left\{x, y, z, w_{1}, w_{2}\right\}$ induces a $\left.C_{5}\right)$ in $G$, then $w \in I[\{x, y, z\}]$ (resp. $\left.w_{1}, w_{2} \in I[\{x, y, z\}]\right)$. Consequently, $\bar{w} \in I\left[w, v_{1}^{t}\right]$ (resp. $\bar{w}_{1}, \bar{w}_{2} \in I\left[\left\{w_{1}, w_{2}, v_{1}^{t}\right\}\right]$ ). Otherwise, if $V(F)=\{x, y, z, w\}$ induces a $2 K_{2}$ in $G$, let $w z \in E(F)$. Recall that $w x, w y \notin E(F)$, then $\bar{w} \in I[\bar{x}, \bar{y}]$. This implies that $w \in I[\bar{w}, z]$. Hence $V(F) \cup V(\bar{F}) \subseteq H(S)$.

Since $\bar{x}, \bar{y} \in H(S) \cap V\left(G_{1}\right)$, and $\bar{x} \bar{y} \notin E(\bar{G})$, we have that $\bigcup_{i=2}^{t} V\left(\bar{G}_{i}\right) \subseteq I[\bar{x}, \bar{y}]$. Next, we show that $V\left(\bar{G}_{1}\right) \subseteq$ $H(S)$. If $F \simeq 2 K_{2}$, as defined $w, z \in V\left(G_{2}\right)$ it is clear that $V\left(\bar{G}_{1}\right) \subseteq I[\bar{w}, \bar{z}]$. Otherwise, $F \subseteq G_{1}$, we let $A \cup B$ be a partition of $V\left(G_{1}\right) \backslash V(F)$, defined as follows.

Let $A=\left\{a \in V\left(G_{1}\right) \backslash V(F): \exists v, v^{\prime} \in N_{F}(a)\right.$ with $\left.v v^{\prime} \notin E(F)\right\}$ and $B=V\left(G_{1}\right) \backslash(V(F) \cup A)$. Trivially, it follows from the definition that $A \subseteq I[V(F)]$. Consequently $\bar{A} \subseteq I\left[A \cup\left\{v_{1}^{t}\right\}\right]$. Remains to show that $B \cup \bar{B} \subseteq$ $H(S)$.

By definitions of $B$ and $F$, every $b \in B$ is such that $N_{F}(b)$ induces a $K_{2}, K_{1}$ or $N_{F}(b)=\emptyset$. This implies that there exist two non-adjacent vertices $\bar{u}, \bar{u}^{\prime} \in N_{\bar{F}}(\bar{b})$. Then $\bar{b} \in I\left[\bar{u}, \bar{u}^{\prime}\right]$ and consequently $\bar{B} \subseteq H(S)$. Since $G_{1}$ is connected and $V\left(G_{1}\right) \cap H(S) \neq \emptyset$, Proposition 3.4 implies that $B \subseteq H(S)$. Hence $V(G \bar{G}) \subseteq H(S)$. Recall that $t+1 \leq|S| \leq t+2$, as desired.

We remark that the bounds $t+1 \leq h(G \bar{G}) \leq t+2$ of Theorem 3.6 are tight. For instance, if $G$ has at least two non-trivial components, then $h(G \bar{G})=t+1$; and if $G$ has exactly one non-trivial component $G_{1}$ that is a butterfly graph (i.e., $V\left(G_{1}\right)=\left\{v_{1}, \ldots, v_{5}\right\}$, and $E\left(G_{1}\right)=\left\{v_{1} v_{2}, v_{3} v_{4}\right\} \cup\left\{v_{i} v_{5}: 1 \leq i \leq 4\right\}$ ), we have $h(G \bar{G})=t+2$. See examples of graphs satisfying those three bounds on Figure 1. The black vertices represent a hull set of each complementary prism. 


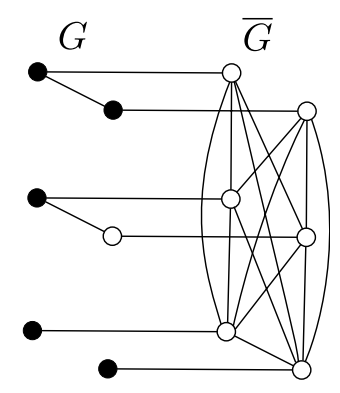

(a) $h(G \bar{G})=t+1=5$.

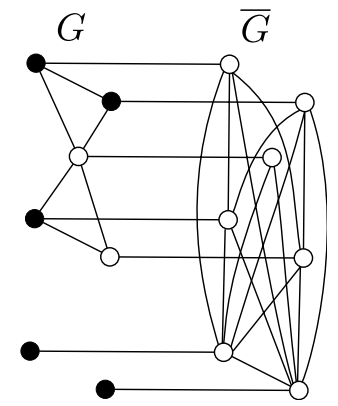

(b) $h(G \bar{G})=t+2=5$.

FIGURE 1. Graphs for the bounds of Theorem 3.6.

\subsection{Split graphs}

We show in this section our results for split disconnected graphs. For a graph $G$ it is known that $G \bar{G}$ is isomporphic to $\bar{G} G$. Then, the next result implies the the hull number for complementary prisms of complete graphs.

Proposition 3.7. Let $G$ be a split graph with $t \geq 2$ connected components. If $G \simeq \bar{K}_{t}$, then $h(G \bar{G})=t$.

Proof. Since $V(G)=\sigma(G \bar{G})$, Lemma 3.1 implies that $h(G \bar{G}) \geq t$. Clearly, $I[V(G)]=H(V(G))=V(G \bar{G})$ and the conclusion follows.

Let $G$ be a disconnected split graph, such that $G \not \bar{K}_{t}$. Since $G$ is a split graph, we know that $2 K_{2}$ is not an induced subgraph of $G$. Then, we assume in this section that $G$ has exactly one nontrivial component and at least one trivial component.

We proceed similarly as done in the work by Castonguay et al. [9] using the following definition and auxiliary result to characterize some convex sets in complementary prisms. For the next, recall Definition 1.1 which expresses how to obtain the component graph.

Definition 3.8. Let $G=(C \cup I, E)$ be a split disconnected graph with exactly one nontrivial component $H$. We define the set $L\left(H_{i}\right)$ of a component $H_{i}$ of $H^{\prime}$ as

$$
L\left(H_{i}\right)=V\left(H_{i}\right) \cup\left(V\left(\bar{H}_{i}\right) \cap \bar{C}\right),
$$

for every $i \in\left[c\left(H^{\prime}\right)\right]$.

Lemma 3.9. Let $G=(C \cup I, E)$ be a split disconnected graph with exactly one nontrivial component $H$. It holds that $V(G \bar{G}) \backslash L\left(H_{i}\right)$ is convex in $G \bar{G}$, for every $i \in\left[c\left(H^{\prime}\right)\right]$.

Proof. Let $G$ be a split disconnected graph obtained from the disjoint union of a nontrivial split connected graph $H$ and a graph $U \simeq t K_{1}$. Let $i \in\left[c\left(H^{\prime}\right)\right]$. To show that $V(G \bar{G}) \backslash L\left(H_{i}\right)$ is convex in $G \bar{G}$, we show that $I[u, v] \cap L\left(H_{i}\right)=\emptyset$, for every $u, v \in V(G \bar{G}) \backslash L\left(H_{i}\right)$. We divide the proof in four cases, considering the possibilities of $u, v \in V(U \bar{U})$ and $u, v \in V(H \bar{H})$ :

(1) $u, v \in V(U)$ or $u \in V(U), v \in V(H) \backslash L\left(H_{i}\right)$;

(2) $u \in V(G) \backslash L\left(H_{i}\right), v \in V(\bar{G}) \backslash L\left(H_{i}\right)$;

(3) $u, v \in V(\bar{G}) \backslash L\left(H_{i}\right)$;

(4) $u, v \in V(H)$.

Trivially, if $u v \in E(G \bar{G})$, then $I[u, v]=\{u, v\}$. Given that $u, v \notin L\left(H_{i}\right)$, clearly $I[u, v]=\{u, v\} \cap L\left(H_{i}\right)=\emptyset$. So, we assume that $u v \notin E(G \bar{G})$. 
Case 1. Let $u, v \in V(U)$ or $u \in V(U)$ and $v \in V(H) \backslash L\left(H_{i}\right)$.

Since $u \bar{u} \bar{v} v$ is the only shortest path between $u$ and $v$, we have that $I[u, v]=\{u, v, \bar{u}, \bar{v}\}$. Notice that $\bar{w} \in$ $V\left(\bar{H}_{i}\right) \cap \bar{C}$ if and only if $w \in V\left(H_{i}\right) \cap C$. Then, given that $u, v \notin V\left(H_{i}\right) \cap C,\left(V\left(\bar{H}_{i}\right) \cap \bar{C}\right) \cap I[u, v]=\emptyset$.

Case 2. Let $u \in V(G) \backslash L\left(H_{i}\right)$ and $v \in V(\bar{G}) \backslash L\left(H_{i}\right)$.

Notice that $I[u, v]=\{u, v\}$, if $v=\bar{u}$, or $I[u, v]=\{u, v, \bar{u}\}$, otherwise. Since $u \notin L\left(H_{i}\right)$, then $\bar{u} \notin L\left(H_{i}\right)$, which implies that $\{u, v, \bar{u}\} \cap L\left(H_{i}\right)=\emptyset$.

Case 3. Let $\bar{u}, \bar{v} \in V(\bar{G}) \backslash L\left(H_{i}\right)$.

Since $\bar{u} \bar{v} \notin E(\bar{G})$, we have that $\bar{u}, \bar{v} \in V(\bar{H})$ and $N_{\bar{G}}(\bar{u}) \cap N_{\bar{G}}(\bar{v}) \subseteq I[\bar{u}, \bar{v}]$. We show that $\left(N_{\bar{G}}(\bar{u}) \cap N_{\bar{G}}(\bar{v})\right) \cap$ $\left(V\left(\bar{H}_{i}\right) \cap \bar{C}\right)=\emptyset$.

Let $\bar{w} \in V\left(\bar{H}_{i}\right) \cap \bar{C}$. Recall that $H$ is split and $u v \in E(H)$, then $|\{u, v\} \cap C| \geq 1$. Since $w \in C$, we have that $\bar{w}$ is not adjacent to both $\bar{u}$ and $\bar{v}$, then $w \notin I[\bar{u}, \bar{v}]$. Consequently, $\left(N_{\bar{G}}(\bar{u}) \cap N_{\bar{G}}(\bar{v})\right) \cap\left(V\left(\bar{H}_{i}\right) \cap \bar{C}\right)=\emptyset$ and $I[\bar{u}, \bar{v}] \cap L\left(H_{i}\right)=\emptyset$.

Case 4. Let $u, v \in V(H)$.

First, if $d_{H}(u, v)=2$, then $I[u, v]=N_{H}(u) \cap N_{H}(v)$. We show that $\left(N_{H}(u) \cap N_{H}(v)\right) \cap V\left(H_{i}\right)=\emptyset$. By contradiction, suppose that there exists $w \in\left(N_{H}(u) \cap N_{H}(v)\right) \cap V\left(H_{i}\right)$. We know that $w \in C$ and $w$ has a neighbor $x \in\{u, v\}$ such that $x \in I$. Since $w \in V\left(H_{i}\right) \cap C$, by Definition 3.8, we have that $x \in V\left(H_{i}\right)$, a contradiction.

Now, consider $d_{H}(u, v)=3$. Then $I[u, v]=\{u, v, \bar{u}, \bar{v}\} \cup\left(N_{H}(u) \cup N_{H}(v)\right)$. Since $u, v \in I$ and $u, v \notin L\left(H_{i}\right)$, we know that $\{\bar{u}, \bar{v}\} \cap L\left(H_{i}\right)=\emptyset$. Finally, with a similar argument to the one presented in previous paragraph, we conclude that $\left(N_{H}(u) \cup N_{H}(v)\right) \cap V\left(H_{i}\right)=\emptyset$.

Lemma 3.9 provides a lower bound for $h(G \bar{G})$, presented in Theorem 3.10.

Theorem 3.10. Let $G$ be a split graph with exactly one nontrivial component $H$ and $t \geq 1$ trivial components. Then $h(G \bar{G}) \geq c\left(H^{\prime}\right)+t$.

Proof. By Lemma 3.9, $V(G \bar{G}) \backslash L\left(H_{i}\right)$, for every $i \in\left[c\left(G^{\prime}\right)\right]$, is a convex set. Thus, Lemma 3.2 implies that every hull set of $G \bar{G}$ must contain at least one vertex from $L\left(H_{i}\right)$, for every $i \in\left[c\left(G^{\prime}\right)\right]$. Since $V\left(H_{i}\right) \cap V\left(H_{j}\right)=\emptyset$, for all $i, j \in\left[c\left(G^{\prime}\right)\right], i \neq j$, each vertex $v \in V(H)$ intersects exactly one $L\left(H_{i}\right)$. This implies that $h(G \bar{G}) \geq c\left(G^{\prime}\right)$.

We know that each vertex $u \in V(G) \backslash V(H)$ is simplicial in $G \bar{G}$. Then Lemma 3.1 implies that a hull set of $G \bar{G}$ contains $u$, for every $u \in V(G) \backslash V(H)$. Since $(V(G) \backslash V(H)) \cap V(H)=\emptyset$, this yields the bound $h(G \bar{G}) \geq c\left(H^{\prime}\right)+t$.

For the upper bounds, we present next our results. For a split graph $H$, we let $c_{\mathrm{nt}}\left(H^{\prime}\right)$ and $c_{\mathrm{t}}\left(H^{\prime}\right)$ denote, respectively, the number of nontrivial and trivial components of its component graph $H^{\prime}$.

Theorem 3.11. Let $G=(C \cup I, E)$ be a split graph with exactly one nontrivial component $H$ and $t \geq 1$ trivial components. If $\max \left\{c_{\mathrm{nt}}\left(H^{\prime}\right), c_{\mathrm{t}}\left(H^{\prime}\right)\right\} \geq 2$, then $h(G \bar{G}) \leq c\left(H^{\prime}\right)+t$.

Proof. Let $G$ be a split disconnected graph obtained from the disjoint union of a nontrivial split connected graph $H$ and a graph $U \simeq t K_{1}$, with $V(U)=\left\{u_{1}, \ldots, u_{t}\right\}$. Let $\mathfrak{I}_{\mathrm{t}}=\left\{i \in\left[c\left(H^{\prime}\right)\right]:\left|V\left(H_{i}\right)\right|=1\right\}, \mathfrak{I}_{\mathrm{nt}}=\left[c\left(H^{\prime}\right)\right] \backslash \mathfrak{I}_{\mathrm{t}}$, and $S=\left\{v_{j} \in V\left(H_{j}\right) \cap C: j \in \mathfrak{I}_{\mathrm{t}}\right\} \cup\left\{v_{k} \in V\left(H_{k}\right) \cap I: k \in \mathfrak{I}_{\mathrm{nt}}\right\} \cup V(U)$. Notice that $|S|=\left|\mathfrak{I}_{\mathrm{t}}\right|+\left|\mathfrak{I}_{\mathrm{nt}}\right|+t=c\left(H^{\prime}\right)+t$. We show that $H(S)=V(G \bar{G})$.

Firstly, since $\bar{u}_{i}$ and $\bar{v}_{j}$ lie in a shortest $\left(u_{i}, v_{j}\right)$-path, for every $i \in[t], j \in\left[c\left(H^{\prime}\right)\right]$, we have that $\bar{S} \subseteq I[S]$. Next, we show that $\bar{I} \subseteq H(S)$, considering either $\left|\mathfrak{I}_{\mathrm{nt}}\right| \geq 2$ or $\left|\mathfrak{I}_{\mathrm{t}}\right| \geq 2$, separately.

If $\left|\mathfrak{I}_{\mathrm{nt}}\right| \geq 2$, we let $i, j \in \mathfrak{I}_{\mathrm{nt}}, i \neq j$. Since $v_{i} x y v_{j}$ induces a $P_{4}$ in $H$, for every $x \in N_{H}\left(v_{i}\right), y \in N_{H}\left(v_{j}\right)$, by applying Proposition 3.5 we have that $N_{H}\left[\left\{v_{i}, v_{j}\right\}\right] \cup \overline{N_{H}\left[\left\{v_{i}, v_{j}\right\}\right]} \subseteq H(S)$. Since $N_{H}\left[v_{i}\right] \cap\left(V\left(H_{k}\right) \cap I\right)=\emptyset$, for every $k \in \mathfrak{I}_{\mathrm{nt}} \backslash\{i\}$, we have that $\overline{V\left(H_{k}\right) \cap I} \subseteq I\left[\overline{N_{H}\left[v_{i}\right]}\right]$. Similarly, since $N_{H}\left[v_{j}\right] \cap\left(V\left(H_{\ell}\right) \cap I\right)=\emptyset$, for every $\ell \in \mathfrak{I}_{\mathrm{nt}} \backslash\{j\}$, we have that $\overline{V\left(H_{\ell}\right) \cap I} \subseteq I\left[\overline{N_{H}\left[v_{j}\right]}\right]$. So, it follows that $\bar{I} \subseteq H(S)$. 
Otherwise, if $\left|\mathfrak{I}_{\mathrm{nt}}\right|=0$, then $H \simeq K_{c\left(H^{\prime}\right)}$ and trivially $I[S]=V(G \bar{G})$. So, we assume that $\left|\mathfrak{I}_{\mathrm{nt}}\right|=1$, which implies, by hypothesis, $\left|\mathfrak{I}_{\mathfrak{t}}\right| \geq 2$. W.l.o.g. let $\mathfrak{I}_{\mathrm{nt}}=\{1\}$ and $\mathfrak{I}_{\mathrm{t}}=\left\{2, \ldots, c\left(H^{\prime}\right)\right\}$. Since $v_{1} x v_{2}$ is a shortest $\left(v_{1}, v_{2}\right)$ path, for every $x \in N_{H}\left(v_{1}\right)$, we have that $N_{H}\left(v_{1}\right) \subseteq I\left[v_{1}, v_{2}\right]$. Recall that $\left|\mathfrak{I}_{\mathfrak{t}}\right| \geq 2$ and $\bar{v}_{2}, \bar{v}_{3} \in H(S)$. Then $\bar{I}=\overline{V\left(H_{1}\right) \cap I} \subseteq I\left[\bar{v}_{2}, \bar{v}_{3}\right] \subseteq H(S)$.

Remains to show that $N_{H}[w] \subseteq H(S)$, for every $w \in I \backslash\left\{v_{i}: i \in \mathfrak{I}_{\mathrm{nt}}\right\}$ and $\bar{C} \in H(S)$.

Claim 1. For every $w \in I \backslash\left\{v_{i}: i \in \mathfrak{I}_{\text {nt }}\right\}, N_{H}[w] \subseteq H(S)$.

Let $i \in \mathfrak{I}_{\text {nt }}$ and $x \in N_{H}\left(v_{i}\right)$. We use induction on $d_{H^{\prime}}(w, x)$, for $w \in I \backslash\left\{v_{i}\right\}$, to show that $N_{H}[w] \subseteq H(S)$. Let $d_{H^{\prime}}(w, x)=1$. Since $w$ lies in a shortest $(x, \bar{w})$-path, we have $w \in I[x, \bar{w}]$. Consequently, $N_{H}(w) \subseteq I\left[w, v_{j}\right]$, for $j \in\left[c\left(H^{\prime}\right)\right] \backslash\{i\}$.

Now, let $d_{H^{\prime}}(w, x)=k$. By induction hypothesis, $N_{H}\left[w^{\prime}\right] \subseteq H(S)$, for $w^{\prime} \in I \backslash\left\{v_{i}\right\}$ such that $d_{H^{\prime}}\left(w^{\prime}, x\right)=$ $k-2$. So, there exists $x^{\prime} \in N_{H}\left(w^{\prime}\right)$ (and then $\left.d_{H^{\prime}}\left(x^{\prime}, x\right)=k-1\right)$ such that $x^{\prime} \in N_{H}(w)$. Thus, $w$ lies in a shortest $\left(x^{\prime}, \bar{w}\right)$-path and $w \in I\left[x^{\prime}, \bar{w}\right]$. Finally, $N_{H}(w) \subseteq I\left[w, v_{j}\right]$, for $j \in\left[c\left(H^{\prime}\right)\right] \backslash\{i\}$.

By Claim 1, we conclude that $V\left(H_{i}\right) \in H(S)$, for every $i \in \mathfrak{I}_{\text {nt }}$. This implies that $\bar{C} \subseteq I\left[C \cup\left\{u_{1}\right\}\right]$, which completes the proof.

Corollary 3.12 follows immediately from Theorems 3.10 and 3.11 .

Corollary 3.12. Let $G=(C \cup I, E)$ be a split graph with exactly one nontrivial component $H$ and $t \geq 1$ trivial components. If $\max \left\{c_{\mathrm{nt}}\left(H^{\prime}\right), c_{\mathrm{t}}\left(H^{\prime}\right)\right\} \geq 2$, then $h(G \bar{G})=c\left(H^{\prime}\right)+t$.

Now, let $\max \left\{c_{\mathrm{nt}}\left(H^{\prime}\right), c_{\mathrm{t}}\left(H^{\prime}\right)\right\} \leq 1$. The case $c_{\mathrm{t}}\left(H^{\prime}\right)=1$ and $c_{\mathrm{nt}}\left(H^{\prime}\right)=0\left(\right.$ resp. $c_{\mathrm{t}}\left(H^{\prime}\right)=0$ and $\left.c_{\mathrm{nt}}\left(H^{\prime}\right)=0\right)$ is not under consideration, since $H \simeq K_{1}$ (resp. $V(H)=\emptyset$ ) implies that $G$ has only trivial components. So, we consider $c_{\mathrm{t}}\left(H^{\prime}\right)=c_{\mathrm{nt}}\left(H^{\prime}\right)=1$ or $c_{\mathrm{t}}\left(H^{\prime}\right)=0$ and $c_{\mathrm{nt}}\left(H^{\prime}\right)=1$.

Theorem 3.13. Let $G=(C \cup I, E)$ be a split graph with exactly one nontrivial component $H$ and $t \geq 1$ trivial components. Let $d=\max \left\{\operatorname{deg}_{H}(v): v \in I\right\}$ and $D=\left\{v \in I: \operatorname{deg}_{H}(y)=d\right\}$. If $\max \left\{c_{\mathrm{nt}}\left(H^{\prime}\right), c_{\mathrm{t}}\left(H^{\prime}\right)\right\} \leq 1$, then

$$
h(G \bar{G})= \begin{cases}|D|+t+1, & \text { if } d=|C|-1 ; \\ t+2, & \text { otherwise. }\end{cases}
$$

Proof. Let $G$ be a split disconnected graph obtained from the disjoint union of a nontrivial split connected graph $H$ and a graph $U \simeq t K_{1}$, with $V(U)=\left\{u_{1}, \ldots, u_{t}\right\}$.

(1) Let $d=|C|-1$. Recall that we consider a partition $V(G)=C \cup I$, in which $C$ is a maximum clique of $G$. Then $c_{\mathrm{t}}\left(H^{\prime}\right)=0$ and $c_{\mathrm{nt}}\left(H^{\prime}\right)=1$ is not considered, since $d=|C|-1$ implies that $C \cup\{y\}$ is a maximum clique in $H$, for some $y \in D$. So, consider $c_{\mathrm{t}}\left(H^{\prime}\right)=c_{\mathrm{nt}}\left(H^{\prime}\right)=1$. Let $H_{\mathrm{t}}$ and $H_{\mathrm{nt}}$ be the trivial and the nontrivial components of $H^{\prime}$, respectively, and $V\left(H_{\mathrm{t}}\right)=\{x\}$.

Suppose that $d=|C|-1$, i.e., $N_{H}(D)=C \backslash\{x\}$. We show that, for every $y \in D, y \in \sigma(G)$ and $\bar{y} \in \sigma(\bar{G})$. Recall that $c_{\mathrm{t}}\left(H^{\prime}\right)=c_{\mathrm{nt}}\left(H^{\prime}\right)=1$. Since $N_{H}[x]=C$, it is clear that $x \in \sigma(G)$. Also, $x \notin N_{H}(I)$ implies that $\bar{x} \in \overline{N_{H}(I)}$. Since $N_{\bar{G}}(\bar{x})=\overline{N_{H}(I)} \cup V(\bar{U})$, which is a clique, it follows that $\bar{x} \in \sigma(\bar{G})$. Further, since $N_{G}(y)=$ $C \backslash\{x\}$, for every $y \in D$, we have that $N_{G}(y)=N_{G}(x)$. This implies that $y \in \sigma(G)$ and $\bar{y} \in \sigma(\bar{G})$, by similar argumentation presented for $x$.

Let $S$ be a hull set of $G \bar{G}$. We know that $V(U)=\sigma(G)$ and, for every $v \in D \cup\{x\}, v \in \sigma(G)$ and $\bar{v} \in \sigma(\bar{G})$. Then, Lemma 3.1 implies that $V(U) \subseteq S$ and Lemma 3.3 implies that $S \cap\{v, \bar{v}\} \neq \emptyset$. This yields the bound $h(G \bar{G}) \geq|V(U)|+|D \cup\{x\}|=|D|+t+1$. Let $S=V(U) \cup D \cup\{x\}$. Since $\bar{S} \subseteq I[S], C \subseteq I[D \cup\{x\}]$, and $\bar{C} \subseteq I\left[C \cup\left\{u_{1}\right\}\right]$, we conclude that $V(G \bar{G})=H(S)$ and $h(G \bar{G}) \leq|D|+t+1$.

(2) Let $d \leq|C|-2$ and $y \in D$. First, notice that $h(G \bar{G}) \geq t+2$ is clear, since $H(S)=S \cup \bar{S} \neq V(G \bar{G})$, when $S$ contains exactly one vertex from each connected component of $G$. So, we prove that $h(G \bar{G}) \leq t+2$. If $c_{\mathrm{t}}\left(H^{\prime}\right)=c_{\mathrm{nt}}\left(H^{\prime}\right)=1$, let $H_{\mathrm{t}}$ and $H_{\mathrm{nt}}$ be the trivial and the nontrivial components of $H^{\prime}$, respectively, and 


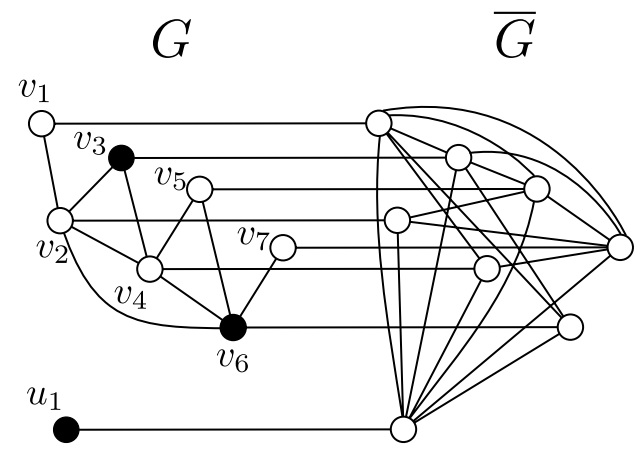

Figure 2. Example of graph $G \bar{G}$ for Case 2 of Theorem 3.13.

$V\left(H_{\mathrm{t}}\right)=\{x\}$. Otherwise, let $x \in C \backslash N_{H}(y)$. We show that $S=\{x, y\} \cup V(U)$ is a hull set of $G \bar{G}$. See in Figure 2 an example of graph $G \bar{G}$ with $c_{\mathrm{t}}\left(H^{\prime}\right)=0$, where $x=v_{6}, y=v_{3}$, and $V(U)=\left\{u_{1}\right\}$.

Trivially, we have that $\bar{S} \subseteq I[S], N_{H}(y) \subseteq I[x, y]$, and $\overline{N_{H}(y)} \subseteq I\left[N_{H}(y) \cup\left\{u_{1}\right\}\right]$.

For every $w \in I \backslash D$, since $\operatorname{deg}_{H}(w)<\operatorname{deg}_{H}(y)$, there exists $x^{\prime} \in N_{H}(y) \backslash N_{H}(w)$. Consequently, $\bar{w}$ lies in a shortest $\left(\bar{x}^{\prime}, \bar{y}\right)$-path, then $\bar{w} \in I\left[\bar{x}^{\prime}, \bar{y}\right]$ and $\overline{I \backslash D} \subseteq H(S)$. Since $H_{\mathrm{nt}}$ is connected, there is a path between $w$ and a vertex in $N_{H}(y) \subseteq H(S)$, for every $w \in I \backslash D$. Then, by similar argumentation to Claim 1 of Theorem 3.11, we conclude that $N_{H}[I \backslash D] \subseteq H(S)$. Consequently $\overline{N_{H}[I \backslash D]} \subseteq I\left[N_{H}[I \backslash D] \cup\left\{v_{1}\right\}\right]$.

Consider first $\left.C \backslash\{x\} \subseteq N_{H}((I \backslash D) \cup\{y\})\right)$. By hypothesis, $d \leq|C|-2$, then there exists $z, z^{\prime} \in C \cup\{x\}$ such that $z, z^{\prime} \notin N\left(y^{\prime}\right)$, for every $y^{\prime} \in D$. Then, $\bar{y}^{\prime} \in I\left[\bar{z}, \bar{z}^{\prime}\right]$. Since $H_{\mathrm{nt}}$ is connected, there exists $z^{\prime \prime} \in N_{H}\left(y^{\prime}\right) \subseteq H(S)$, then $y \in I\left[z^{\prime \prime}, \bar{y}^{\prime}\right]$. Hence $H(S)=V(G \bar{G})$.

Now, consider $\left.C \backslash\{x\} \nsubseteq N_{H}((I \backslash D) \cup\{y\})\right)$. Let $v \in C \backslash\{x\}$ such that $\left.v \notin N_{H}((I \backslash D) \cup\{y\})\right)$. Observe that we do not know yet whether $v \in H(S)$. Since $H_{\mathrm{nt}}$ is connected, $c_{\mathrm{t}}\left(H^{\prime}\right) \leq 1$ and $v \neq x$, we have that $N_{H}(v) \cap(D \backslash\{y\}) \neq \emptyset$. Let $y^{\prime} \in N_{H}(v) \cap(D \backslash\{y\})$. Since $\operatorname{deg}_{H}\left(y^{\prime}\right)=\operatorname{deg}_{H}(y)=d$ and $v \in N_{H}\left(y^{\prime}\right) \backslash N_{H}(y)$, we have that $\left|N_{H}(y) \cap N_{H}\left(y^{\prime}\right)\right| \leq d-1$. Then, there exists $z \in N_{H}(y) \backslash N_{H}\left(y^{\prime}\right)$. This implies that $\bar{y}^{\prime} \in I[\bar{y}, \bar{z}]$. Again, since $H_{\mathrm{nt}}$ is connected, there is a path between $y^{\prime}$ and a vertex in $N_{H}((I \backslash D) \cup\{y\}) \subseteq H(S)$. Consequently $N\left[y^{\prime}\right] \subseteq H(S)$ and finally $H(S)=V(G \bar{G})$.

Remark 3.14. Let $G=(C \cup I, E)$ be a split graph with exactly one nontrivial component $H$ and $t \geq 1$ trivial components. In our preliminary work [11], we show that if $\operatorname{diam}(H) \leq 3$, then $h(G \bar{G}) \leq h(H)+t$, which is not tight. Let, for instance, $H$ be the graph arising from a path $P_{k}: v_{1} v_{2} \ldots v_{k}$, for some odd $k \geq 5$, by adding $v_{i} v_{j} \in E(H)$, for all even $i, j \in\{1, \ldots, k\}$. Theorem 3.5 in [11] establish that $h(G \bar{G}) \leq h(H)+t=\left\lceil\frac{k}{2}\right\rceil+t$ whereas $h(G \bar{G})=t+2$ (Case 2 of Thm. 3.13). See an example of graph $G$ with $k=7$ in Figure 2. A hull set of $G \bar{G}$ is represented by the vertices in black. It is easy to see that $h(H)+t=\left|\left\{v_{1}, v_{3}, v_{5}, v_{7}\right\}\right|+1=5$ and $h(G \bar{G})=3$.

\subsection{Further results}

We end Section 3 with some further corollaries. We remark that the recognition of complementary prisms can be done in $O\left(n^{6}\right)$ time [8]. So, for $G \bar{G}$, input graph for Corollary 3.15, we may assume that $G$ is known. In addition, for a graph $G$ with $n$ vertices and $m$ edges and a set $S \subseteq V(G)$, computing $H(S)$ can be done in $O(n m)$ time [15]. Clearly, given that $|V(G \bar{G})|=n$, we know that $|E(G \bar{G})|=\frac{\frac{n}{2}\left(\frac{n}{2}-1\right)}{2}+\frac{n}{2}=\frac{n^{2}}{8}+\frac{n}{4}=\Theta\left(n^{2}\right)$. Then, for $S \subseteq V(G \bar{G}), H(S)$ can be computed in $O\left(n^{3}\right)$ time.

Corollary 3.15. Given a complementary prism $G \bar{G}$, for some graph $G$, one can compute the geodetic hull number of $G \bar{G}$ in $O\left(n^{6}\right)$ time. 
Proof. Let $G \bar{G}$ be a complementary prism, for some graph $G,|V(G \bar{G})|=n$, and $|E(G \bar{G})|=m=\Theta\left(n^{2}\right)$. First, consider that $G$ is a nonsplit graph. We remark that recognizing a split graph can be done in linear time [22]. We have that $2 \leq h(G \bar{G}) \leq 3$ [9], if $G$ and $\bar{G}$ are both connected, or $t+1 \leq h(G \bar{G}) \leq t+2$ (Thm. 3.6), otherwise. In the former, it is obvious that, by choosing every 2-element and 3-element subset of $V(G \bar{G})$ is possible to find a minimum hull set of $G \bar{G}$ in time $\left(\left(\begin{array}{l}n \\ 2\end{array}\right)+\left(\begin{array}{l}n \\ 3\end{array}\right)\right) \cdot O\left(n^{3}\right)=O\left(n^{6}\right)$.

For the latter, we need to compute $c_{\mathrm{nt}}(G)$ and $c_{\mathrm{t}}(G)$. By performing a depth first search (DFS) we can find the connected components of $G$ to compute their orders, spending $O(|V(G)|+|E(G)|)=O\left(\frac{n}{2}+\left(\frac{n}{2}\right)^{2}\right)=O\left(n^{2}\right)$ time. If $c_{\mathrm{nt}}(G) \geq 2$, then in $O(1)$ we return $h(G \bar{G})=t+1$ (Thm. 3.6). Otherwise, as we know that $c_{\mathrm{nt}}(G)=1$ and $c_{\mathrm{t}}(G) \geq 1$, we let $S$ formed by the vertices of every trivial component of $G$ and for the nontrivial component $H$, we choose every 2-element and 3-element subset of $V(H)$. With this, it is possible to find a minimum hull set of $G \bar{G}$ in time $\left(\left(\begin{array}{cc}|V(H)| \\ 2\end{array}\right)+\left(\begin{array}{c}|V(H)| \\ 3\end{array}\right)\right) \cdot O\left(n^{3}\right)=O\left(n^{3}\right) \cdot O\left(n^{3}\right)=O\left(n^{6}\right)$.

Now, let $G$ be a split graph. First, we discuss on computing component graphs. We consider the partition of $V(G)=C \cup I$ such that $C$ is a maximum clique. Such a partition can be found in linear time [10]. As done in [9], and also in this paper, we need to compute the component graph $G^{\prime}$ of $G$, or $H^{\prime}$ of the nontrivial component $H$ of $G$. So, let $F^{\prime} \in\left\{G^{\prime}, H^{\prime}\right\}$. We obtain the component graph $F^{\prime}$ by simply removing the edges between vertices in $C$, which runs in $O\left(|C|^{2}\right)=O\left(n^{2}\right)$ time. Then, computing $c\left(F^{\prime}\right), c_{\mathrm{nt}}\left(F^{\prime}\right)$, and $c_{\mathrm{t}}\left(F^{\prime}\right)$ run in $O\left(n^{2}\right)$ time. The values $c\left(F^{\prime}\right), c_{\mathrm{nt}}\left(F^{\prime}\right)$, and $c_{\mathrm{t}}\left(F^{\prime}\right)$ are required for the next steps.

Consider that $G$ and $\bar{G}$ are connected. Castonguay et al. [9] show that if $\max \left\{c_{\mathrm{nt}}\left(G^{\prime}\right), c_{\mathrm{t}}\left(G^{\prime}\right), c_{\mathrm{nt}}\left(\bar{G}^{\prime}\right), c_{\mathrm{t}}\left(\bar{G}^{\prime}\right)\right\} \geq$ 2 , then $h(G \bar{G})=\max \left\{c\left(G^{\prime}\right), c\left(\bar{G}^{\prime}\right)\right\}$ and $2 \leq h(G \bar{G}) \leq 3$, otherwise. Clearly, computing if some value in $\left\{c_{\mathrm{nt}}\left(G^{\prime}\right), c_{\mathrm{t}}\left(G^{\prime}\right), c_{\mathrm{nt}}\left(\bar{G}^{\prime}\right), c_{\mathrm{t}}\left(\bar{G}^{\prime}\right)\right\}$ is greater than 2 and computing the maximum between $c\left(G^{\prime}\right)$ and $c\left(\bar{G}^{\prime}\right)$ can be done in linear time. Furthermore, again by choosing every 2-element and 3-element subset of $V(G \bar{G})$, is possible to find a minimum hull set of $G \bar{G}$ in $O\left(n^{6}\right)$ time.

Now, let $G$ be a disconnected graph with one nontrivial component $H$ and $t \geq 1$ trivial components. Such components of $H^{\prime}$ and $t$ can be found in $O\left(n^{2}\right)$. By Corollary 3.12, $h(G \bar{G})=c\left(H^{\prime}\right)+t$ if $\max \left\{c_{\mathrm{nt}}\left(H^{\prime}\right), c_{\mathrm{t}}\left(H^{\prime}\right)\right\} \geq$ 2. Such a condition can be checked in $O\left(c_{\mathrm{nt}}\left(H^{\prime}\right)+c_{\mathrm{t}}\left(H^{\prime}\right)\right)=O(n)$ time. Otherwise, $h(G \bar{G})=|D|+t+1$, if $d=|C|-1$ or $h(G \bar{G})=t+2$, if $d<|C|-1$ (recall Thm. 3.13). So, we need to compute $d=\max \left\{\operatorname{deg}_{H}(v): v \in I\right\}$ and $D=\left\{v \in I: \operatorname{deg}_{H}(y)=d\right\}$, which can be obtained in $O(n)$ by the degree sequence of $G$ [22]. For the rest, checking whether $d=|C|-1$ runs in constant time.

It is easy to observe that the overall running time for determining $h(G \bar{G})$, for any graph $G$, is of order $O\left(n^{6}\right)$ and the conclusion holds.

Next, we have the following Corollary 3.16 on $h(G \bar{G})$ when $G$ is a tree. It has been first proved by [11], and here we leave an alternative proof. A star $S_{n}$ is the complete bipartite graph $K_{1, n}$.

Corollary 3.16. [11] Let $T$ be a tree on $n$ vertices. It holds that $h(T \bar{T})=n$ if $T$ is a star, or $h(T \bar{T})=2$, otherwise.

Proof. If $T$ is a star, we have that $\bar{T} \simeq K_{1} \cup K_{n-1}$. Since $c_{\mathrm{t}}\left(K_{n-1}\right)=n-1$, Corollary 3.12 implies that $h(T \bar{T})=$ $n$. Otherwise, we have that $n \geq 4$ and both $T$ and $\bar{T}$ are connected. If $T$ is split, $c_{\mathrm{nt}}\left(T^{\prime}\right)=2$, then Corollary 3.16 of [9] implies that $h(T \bar{T})=2$. If $T$ is not split, then $2 K_{2}$ is an induced subgraph of $T$ and the result $h(T \bar{T})=2$ holds by Theorem 3.8 of [9].

A cograph is a graph with no induced $P_{4}$. Since a nontrivial cograph $G$ is connected if and only if $\bar{G}$ is disconnected [13], we can update Theorem 3.9 of [11] to obtain the following.

Corollary 3.17. Let $G$ be a connected cograph. Then:

(i) $h(G \bar{G})=c_{t}(\bar{G})$, if $c_{n t}(\bar{G})=0$;

(ii) $c_{t}(\bar{G})+2 \leq h(G \bar{G}) \leq|D|+c_{t}(\bar{G})+1$, if $c_{n t}(\bar{G})=1$;

(iii) $h(G \bar{G})=c(\bar{G})+1$, if $c_{n t}(\bar{G}) \geq 2$. 
Proof. The proof of (i), (ii) and (iii) follows, respectively, by Proposition 3.7, Theorem 3.13, and Theorem 3.6.

\section{ConCluding REMARKS}

Whereas computing the hull number in geodetic convextiy is NP-hard for arbitrary graphs, we have shown that the problem is polynomial time solvable for complementary prisms. In such a class of graphs, determining the parameter is also polynomial time solvable in $P_{3}$-convexity [18]. This raises the possibility of finding other graph convexities in which hull number is hard for general graphs, but can be computed in polynomial time when restricted to complementary prisms.

\section{REFERENCES}

[1] J. Akiyama and F. Harary, A graph and its complement with specified properties I: Connectivity. Int. J. Math. Math. Sci. 2 (1979) 223-228.

[2] M. Albenque and K. Knauer, Convexity in partial cubes: the hull number, in LATIN 2014: Theoretical Informatics. Springer (2014) 421-432.

[3] J. Araujo, V. Campos, F. Giroire, N. Nisse, L. Sampaio and R. Soares, On the hull number of some graph classes. Theor. Comput. Sci. 475 (2013) 1-12.

[4] J. Araujo, G. Morel, L. Sampaio, R. Soares and V. Weber, Hull number: $P_{5}$-free graphs and reduction rules. Discr. Appl. Math. 210 (2016) 171-175.

[5] S. Bessy, M.C. Dourado, L.D. Penso and D. Rautenbach, The geodetic hull number is hard for chordal graphs. SIAM J. Discr. Math. 32 (2018) 543-547.

[6] B. Bollobás, The Art of Mathematics: Coffee Time in Memphis. Cambridge University Press (2006).

[7] P.P. Camargo, U.S. Souza and J.R. Nascimento, Remarks on k-clique, k-independent set and 2-contamination in complementary prisms. Int. J. Found. Comput. Sci. (2021) 1-16.

[8] M.R. Cappelle, L. Penso and D. Rautenbach, Recognizing some complementary products. Theor. Comput. Sci. 521 (2014) $1-7$.

[9] D. Castonguay, E.M.M. Coelho, H. Coelho and J.R. Nascimento, On the geodetic hull number for complementary prisms II. RAIRO-Oper. Res. 55 (2021) S2403-S2415.

[10] V. Chvátal, C.T. Hoàng, N.V.R. Mahadev and D. De Werra, Four classes of perfectly orderable graphs. J. Graph Theory 11 (1987) 481-495.

[11] E.M.M. Coelho, H. Coelho, J.R. Nascimento and J.L. Szwarcfiter, On the geodetic hull number of complementary prisms. Preprint arXiv:1807.08295 (2018).

[12] E.M.M. Coelho, H. Coelho, J.R. Nascimento and J.L. Szwarcfiter, On the $P_{3}$-hull number of some products of graphs. Discr. Appl. Math. 253 (2019) 2-13.

[13] D. Corneil, H. Lerchs and L. Burlingham, Complement reducible graphs. Discr. Appl. Math. 3 (1981) $163-174$.

[14] P. Domingos and M. Richardson, Mining the network value of customers. In Proceedings of the Seventh ACM SIGKDD International Conference on Knowledge Discovery and Data Mining, KDD '01. ACM, New York, NY, USA (2001) $57-66$.

[15] M.C. Dourado, J.G. Gimbel, J. Kratochvíl, F. Protti and J.L. Szwarcfiter, On the computation of the hull number of a graph. Discr. Math. 309 (2009) 5668-5674.

[16] M.C. Dourado, L.D. Penso and D. Rautenbach, On the geodetic hull number of $P_{k}$-free graphs. Theor. Comp. Sci. 640 (2016) $52-60$.

[17] P.A. Dreyer and F.S. Roberts, Irreversible k-threshold processes: Graph-theoretical threshold models of the spread of disease and of opinion. Discr. Appl. Math. 157 (2009) 1615-1627.

[18] M.A. Duarte, L. Penso, D. Rautenbach and U. dos Santos Souza, Complexity properties of complementary prisms. J. Combinat. Optim. (2015) 1-8.

[19] M.G. Everett and S.B. Seidman, The hull number of a graph. Discr. Math. 57 (1985) 217-223.

[20] A. Farrugia, Self-complementary graphs and generalisations: a comprehensive reference manual. University of Malta (1999).

[21] S. Foldes and P.L. Hammer, Split graphs, in Proceedings 8th Southeastern Conference on Combinatorics, Graph Theory and Computing, Louisiana State University, Baton Rouge, LA (1977) 311-315.

[22] P.L. Hammer and B. Simeone, The splittance of a graph. Combinatorica 1 (1981) 275-284.

[23] T.W. Haynes, M.A. Henning, P.J. Slater and L.C. van der Merwe, The complementary product of two graphs. Bull. Inst. Combinator. Appl. 51 (2007) 21-30.

[24] M.M. Kanté and L. Nourine, Polynomial time algorithms for computing a minimum hull set in distance-hereditary and chordal graphs. SIAM J. Discr. Math. 30 (2016) 311-326.

[25] H. Karami, S.M. Sheikholeslami, A. Khodkar and D.B. West, Connected domination number of a graph and its complement. Graphs Combinator. 28 (2012) 123-131. 
[26] D. Peleg, Local majorities, coalitions and monopolies in graphs: a review. Theor. Comput. Sci. 282 (2002) $231-257$.

[27] S.-j. Xu, Some parameters of graph and its complement. Discr. Math. 65 (1987) 197-207.

\section{Subscribe to Open (S20) A fair and sustainable open access model}

This journal is currently published in open access under a Subscribe-to-Open model (S2O). S2O is a transformative model that aims to move subscription journals to open access. Open access is the free, immediate, online availability of research articles combined with the rights to use these articles fully in the digital environment. We are thankful to our subscribers and sponsors for making it possible to publish this journal in open access, free of charge for authors.

\section{Please help to maintain this journal in open access!}

Check that your library subscribes to the journal, or make a personal donation to the $\mathrm{S} 2 \mathrm{O}$ programme, by contacting subscribers@edpsciences.org

More information, including a list of sponsors and a financial transparency report, available at: https://www.edpsciences.org/en/maths-s2o-programme 